\title{
Multivariate Analysis for Operative Mortality in Obstructive Prosthetic Valve Dysfunction Due to Pannus and Thrombus Formation
}

\author{
Mehmet Erdem ToKer, ${ }^{1}$ MD, Ercan Eren,,${ }^{1} \mathrm{MD}$, Mehmet BalKanay, ${ }^{1} \mathrm{MD}$, \\ Kaan KIRALI, ${ }^{1}$ MD, Mehmet YANARTAŞ, ${ }^{1} \mathrm{MD}$, Ahmet ÇALIŞKAN, ${ }^{1} \mathrm{MD}$, \\ Mustafa GÜLER, ${ }^{1} \mathrm{MD}$, and Cevat YAKUT, ${ }^{1} \mathrm{MD}$
}

\section{SUMMARY}

The objective of the present study was to investigate the risk factors for early hospital mortality in reoperations performed for obstructive prosthetic valve dysfunction. Between January 1994 and April 2005, 63 patients underwent reoperation for obstructive prosthetic valve dysfunction. The mean age of the patients was $40.3 \pm 12.8$ years. The mitral valve was replaced in $47(74.6 \%)$ patients, the aortic valve in $6(9.5 \%)$ patients, and both valves in $10(15.9 \%)$ patients. Forty-three $(68.2 \%)$ patients underwent emergency reoperations.

Early hospital mortality occurred in $13(20.6 \%)$ patients. The ethiology of the valve dysfunction was pannus formation in $45(71.4 \%)$ patients and thrombus formation in 18 (28.6\%). Pannus and thrombus were localized at the atrial side of the prosthetic valve in $15(23.9 \%)$ patients, at the ventricular side in $13(20.6 \%)$, and at both sides in $35(55.5 \%)$. Inadequate anticoagulation was diagnosed in 28 of 63 (44.4\%) patients. The mean INR level in these 28 patients was $1.43 \pm 0.24$. In multivariate analysis, the only risk factor for early hospital mortality was left ventricular ejection fraction $(P=0.015$; Odds: 0.000 , 95\% CI: 0.000-0.043).

It is concluded reoperations for prosthetic valve dysfunction have a high mortality rate. This study revealed that left ventricular dysfunction is the major determinant of surgical mortality in patients requiring reoperation for valve dysfunction due to pannus or thrombus. (Int Heart J 2006; 47: 237-245)

Key words: Pannus, Thrombus, Prosthetic valve dysfunction

INTRACARDIAC mechanical prosthetic valve replacement was first introduced in 1960 and many improvements in design and structural properties have increased the hemodynamic performance and durability since then. However, during the last 4 decades obstructive prosthetic valve dysfunction (PVD) has continued to be a potential complication with a high mortality risk because of the thrombogenic potential and need for lifetime anticoagulation therapy. Obstruc-

From the ${ }^{1}$ Department of Cardiovascular Surgery, Kosuyolu Heart and Research Hospital, İstanbul, Turkey.

Address for correspondence: Mehmet Erdem Toker, MD, Department of Cardiovascular Surgery, Kosuyolu Heart and Rescarch Hospital, Yüksek Ticaretliler Sitesi Faikbeymescidi Sokak No:5 A Blok Daire: 17 Acıbadem, Kadıköy Istanbul, Turkey. Received for publication August 30, 2005.

Revised and accepted November 24, 2005. 
tive PVD is frequently related to thrombus formation due to inadequate anticoagulation, however, it can also be related to pannus formation due to overgrowth of fibrous tissue. ${ }^{1)}$ Obstructive PVD has other causes, such as patient-valve mismatch or failure of hemodynamic performance, particularly in first generation mechanical valves. A lack of cooperation between the patient and cardiologist based on information is the main factor in PVD all over the world.

For the group of patients involved in our study, the terms "mechanical valve thrombus" or "stuck valve" are insufficient because the underlying factors could be diverse. Therefore, the term PVD is used only in patients undergoing reoperation for pannus or thrombus, or both, excluding other etiological factors.

PVD due to pannus or thrombus is related mainly to 3 factors: 1) the surface characteristics of the prosthetic valve related with the design and the material used, 2) blood flow which depends on cardiac output, and 3) turbulance, stagnation, and the properties of the patient's blood due to hypercoagulability. ${ }^{2)}$

In our study, patients reoperated on between January 1994 and April 2005 with a diagnosis of PVD were investigated for preoperative properties, mortality rates, and the type of mechanical valve used. Factors affecting mortality were examined using multivariate analysis.

\section{Methods}

Between Janary 1994 and April 2005, 5802 patients were operated on in our department for mechanical prosthetic valve replacement. This number includes valve replacements with or without coronary artery bypass or other cardiac procedures. Sixty-three $(1.08 \%)$ of these patients were reoperated on with the diagnosis of PVD due to thrombus, pannus, or both. Patients reoperated on for valve insufficiency following reconstruction, bioprosthesis dysfunction, or prosthetic valve endocarditis with pannus or thrombus were excluded. Information about the patients was investigated retrospectively.

The mean age was $40.3 \pm 12.8$ years (range, 18 to 71 ), and there were 42 $(66.7 \%)$ females and $21(33.3 \%)$ males. Forty-seven $(74.6 \%)$ patients were operated on for mitral, $6(9.5 \%)$ for aortic, and 10 (15.9\%) for both aortic and mitral PVD. The mean time interval between 2 operations was $58.9 \pm 56.1$ months (1252) (Table I).

Forty-one $(65.1 \%)$ patients were operated on under emergency conditions, 4 $(6.3 \%)$ patients had accompanying systemic diseases (1 chronic renal insufficiency, 3 epilepsy), 5 (7.9\%) patients were pregnant, and 27 (42.9\%) had preoperative atrial fibrillation. Five (11.9\%) received preoperative streptokinase therapy. Eleven (17.5\%) patients had a past cerebrovascular event (CVE). The diagnosis was confirmed by transthoracic echocardiography (TTE ) in 29 (46\%) 
patients, transesophageal echocardiography (TEE) in $30(47.6 \%)$, and cineangiography in $4(6.3 \%)$ (Table I).

The right femoral artery was first explored in all cases. In emergent cases the right femoral artery and vein were cannulated. A median sternotomy was performed using an oscillating saw. The ascending aorta was cannulated in the elective cases. Venous cannulation was performed bicavally or from the superior vena cava together with the right femoral vein. A vent cannula was inserted into the right upper pulmonary vein for left ventricular venting. Antegrade or combined antegrade and retrograde isothermic blood cardioplegia was used for myocardial protection; $30^{\circ} \mathrm{C}$ systemic hypothermy was maintained. In some cases, where the mitral valve was exposed following the right atriotomy, the transseptal approach was preferred due to severe adhesions.

A commercial statistical software package (SPSS for Windows, version 10.0, SPSS Inc, Chicago) was used for data analysis. Results are presented as the mean \pm standard deviation. Univariate analysis was performed using a $t$ test or Mann-Whitney U test for continuous variables and the chi-square or Fisher exact test for categoric variables when appropriate. Risk factors affecting mortality were analyzed by univariate analysis, which was followed by multivariate stepwise logistic regression analyses. The factors that reached significance were analyzed by multivariate analysis.

Table I. Preoperative Patient Data $(n=63)$

\begin{tabular}{ll}
\hline Mean age (years) & $40.3 \pm 12.8$ \\
Female & $42(66.7 \%)$ \\
Emergency & $43(68.2 \%)$ \\
Interval between the 2 operations (months) & $58.9 \pm 56.1$ \\
Systemic disease & $4(6.3 \%)$ \\
Pregnancy & $5(7.9 \%)$ \\
Streptokinase treatment & $5(7.9 \%)$ \\
Preoperative atrial fibrillation & $27(42.9 \%)$ \\
Mean mitral gradient (mmHg) & $17.07 \pm 12.01(10-45)$ \\
Mean aortic gradient (mmHg) & $86.3 \pm 28.3(70-110)$ \\
Transthoracic echocardiography & $29(46.1 \%)$ \\
Transesophageal echocardiography & $30(47.6 \%)$ \\
Cineangiography & $4(6.3 \%)$ \\
Preoperative cerebrovascular event & $11(17.5 \%)$ \\
Mitral prosthetic valve dysfunction & $47(74.6 \%)$ \\
Aortic prosthetic valve dysfunction & $6(9.5 \%)$ \\
Mitral and aortic prosthetic valve dysfunction & $10(15.9 \%)$ \\
Mean INR level * (28 patients) & $1.43 \pm 0.24$ \\
Mean left atrium diameter (cm) & $4.49 \pm 1.3(2.5-6.8)$ \\
Bileaflet valves & $37(58.7 \%)$ \\
Monoleaflet valves & $26(41.3 \%)$ \\
&
\end{tabular}

*Including 28 patients whose INR levels were lower than 2. 


\section{ReSults}

The hospital mortality rate was $20.6 \%$ (13 patients). The cause of death was low cardiac output (LCO) syndrome in 8 patients, CVE in 3 patients, and multiorgan failure and sepsis after prolonged intubation due to severe chronic obstructive pulmonary disease (COPD) in 2 patients. Renal insufficiency requiring dialysis developed in 2 patients and monoplegia developed in one patient. The mean aortic cross clamp (ACC) time was $85.5 \pm 36.4$ (29-190) minutes and total perfusion time (TPT) was $135.3 \pm 68.73$ minutes. Pannus was found in $45(71.4 \%)$ patients and thrombus in $18(28.6 \%)$. Pannus or thrombus was located on the atrial side of the prosthesis in 15 (23.9\%), on the ventricular side in 13 (20.6\%), and both in 35 patients $(55.5 \%)$ (Table II).

Table II. Intraoperative Patient Data $(n=63)$

\begin{tabular}{ll}
\hline Mortality & $13(20.63 \%)$ \\
Aortic cross clamp time (min) & $85.5 \pm 36.4$ \\
Total perfusion time (min) & $135.3 \pm 68.73$ \\
Pannus & $45(71.4 \%)$ \\
Thrombus & $18(28.6 \%)$ \\
Atrial location & $15(23.9 \%)$ \\
Ventricular location & $13(20.6 \%)$ \\
Both atrial and ventricular location & $35(55.5 \%)$ \\
\hline
\end{tabular}

Table III. Univariate Analysis of Risk Factors for InHospital Mortality

\begin{tabular}{lc}
\hline Factor & $P^{*}$ \\
\hline ACCT & 0.202 \\
TPT & 0.079 \\
Preoperative atrial fibrillation & 0.760 \\
Preoperative renal insufficiency & 0.673 \\
Preoperative CVE & 0.062 \\
Emergency operation & 0.514 \\
PAP > 50 mmHg & 0.635 \\
Gender (female) & 0.187 \\
Tilting disk & 0.532 \\
LVESD & 0.006 \\
LVEDD & 0.001 \\
LVEF & 0.001 \\
NYHA Class & \\
$\quad$ 1-2 & 0.962 \\
\end{tabular}

ACCT indicates aortic cross clamping time; TPT, total perfusion time; CVE, cerebrovascular event; PAP, pulmonary artery systolic pressure; LVESD, left ventricular end systolic diameter; LVEDD, left ventricular end diastolic diameter; LVEF, left ventricular ejection fraction; and NYHA, New York Heart Association $* P<0.05$. 
Table IV. Predictors of Early Hospital Mortality in Multivariate Analysis

\begin{tabular}{llll}
\hline Risk factor & Odds ratio & CI (95\%) & $P^{*}$ \\
\hline LVESD & 0.381 & $0.040-3.604$ & 0.400 \\
LVEDD & 6.046 & $0.994-36.790$ & 0.051 \\
LVEF & 0.000 & $0.000-0.043$ & 0.015 \\
\hline LVESD indicates left ventricular end systolic \\
diameter; LVEDD, left ventricular end diastolic \\
diameter; and LVEF, left ventricular ejection frac- \\
tion. \\
$* P<0.05$.
\end{tabular}

Inadequate anticoagulation levels (INR < 2) were detected in 28 of the patients $(28 / 63 ; 44.4 \%)$. The mean INR level in these 28 patients was $1.43 \pm 0.24$ (Table I).

Prosthetic valve dysfunction was seen in 37 (58.7\%) patients with bileaflet mechanical valves and in $26(41.2 \%)$ patients with monoleaflet mechanical valves (Table I). In univariate analysis, the only factors affecting early hospital mortality were left ventricular end systolic diameter (LVESD) $(P=0.006)$, left ventricular end diastolic diameter (LVEDD) $(P=0.001)$, and left ventricular ejection fraction (LVEF) $(P=0.001)$ (Table III). In multivariate analysis, the only factor affecting early hospital mortality was left ventricular ejection fraction (LVEF) $(P=0.015$; Odds: 0.000, 95\% CI: 0.000-0.043) (Table IV).

\section{DISCUSSION}

Endocardial endothelium is a structural and mechanical barrier between blood and subendothelial tissue which is highly thrombogenic. ${ }^{3)}$ Normal endocardium neither activates the coagulation cascade nor intervenes in the adhesion of thrombocytes or other blood components. ${ }^{4)}$ Also, the cellular morphology of the endocardium is very sensitive to the characteristics of blood flow. ${ }^{5)}$ Besides the normal physiological laminary flow, the maintenance of transprosthetic nonphysiological turbulent flow results in an increase in endothelial cycling. This cycling causes the loss of thromboresistant properties due to injury and dysfunction. ${ }^{6}$ )

Thrombus formation is due to the interaction of cellular and plasmic components of blood with endocardium which is surgically injured and has structural and metabolic changes due to variable blood flow and the surface of the mechanical valve that has thrombogenic properties. ${ }^{4,5)}$ Thrombotic and thromboembolic events seen in the first months following surgery could be explained by an increased intrinsic coagulation system due to the nonendothelialized contact surface of the surrounding cuff of the mechanical valve and the presence of the 
injured tissue. ${ }^{7)}$

In our centre, INR levels are 2 to 3.5 in patients undergoing primary mitral or aortic mechanical valve replacement, and 3 to 4.5 in those undergoing double mechanical valve replacement. Following open heart surgery, all patients are examined at our centre after 2 months. However, the vast majority of the patients are referred to our hospital from distant regions of the country. Therefore, it is impossible to say exactly whether anticoagulation is sufficient in all patients in the follow-up period after 2 months. Inadequate anticoagulation levels were detected in $44.4 \%$ (28/63) of the patients in our study (Table I).

Thrombus is a condition stimulated by thrombocyte activation and factors such as the adequacy of anticoagulation, cardiac rythym, and blood flow characteristics. ${ }^{1,8)}$ Pannus formation, which is an overgrowth of fibrous tissue, is an inflammatory reaction against a foreign body blocking the disc by invading the valve orifice. Histological investigations have showed that there are collagen and elastic tissues containing endothelial cells, chronic inflammatory cells, and myofibroblasts in pannus tissue..$^{9)}$ Pannus tissue can grow very slowly and may cause obstruction 10-15 years after mechanical valve replacement. ${ }^{7)}$ A thrombus layer can be secondarily formed on pannus. In our study, we detected thrombus in 18 patients and pannus in 45 . However, in a series of 112 cases described by Deviri and colleagues, ${ }^{1)}$ pannus was seen in $10.7 \%$, pannus and thrombus in $11.6 \%$, and thrombus in $77.7 \%$.

Thrombus or pannus was detected in the mitral position in 47 patients, in the aortic position in 6 , and in both in 10 . Intraoperatively, they were located on the atrial side of the mitral valve in 15 patients, on the ventricular side in 13 patients, and on both the atrial and ventricular sides in 35 patients. According to studies in the literature, the most common localization site was the atrial side of the mitral valve. Turbulent flow, recirculation areas, and transprosthetic flow conditions such as different local pressure areas result in a high risk of thrombus in an atrioventricular position compared to the aortic valve. ${ }^{10)}$ Lower pressure areas cause formation of more fibrous tissue. Abnormal formation of the fibrous tissue, which plays a role in surrounding cuff epithelialization according to the location of the prosthetic valve, is seen most commonly on the atrial side of the tricuspid valve followed by the atrial side of the mitral valve and ventricular side of the tricuspid valve due to the adjacent flow condition. Because of the high arterial pressure, a thin layer of endothelial tissue formation occurs on the ventricular side of the mitral valve and both sides of the aortic valve. These findings can explain why thrombotic events are more common in the tricuspid and mitral positions compared to the aortic position. ${ }^{2,11,12)}$

Forty-one patients underwent emergency surgery. TEE could be performed in 30 patients. Some patients were operated on following only TTE or cinean- 
giography because of their hemodynamic instability.

Five of our patients were pregnant, 4 had systemic disorders, and 28 had inadequate anticoagulation. Recently, Durrleman, et $a l^{13)}$ stated that an inadequate level of anticoagulation is the most important factor involved in the pathogenesis of prosthetic valve thrombosis. All factors transiently or permanently stimulating the coagulation factors and thrombocyte aggregation increase the risk of local thrombus. The level of coagulability is determined by the instant effects of procoagulant and anticoagulant factors. Inadequate anticoagulation, loss of atrial contraction, oral contraceptives, systemic drugs like estrogen, systemic diseases such as malignant tumors, a defect on the surface of a mechanical prosthetic valve, and chronic endothelial injury due to variable blood flow can all cause local hypercoagulability. ${ }^{14-17)}$ Thrombus on the mechanical valve may often lead to congestive heart failure. However, it may also lead to CVE, which was seen in $17.5 \%(11 / 63)$ of our patients. Rarely, it may cause vasospastic angina due to coronary thromboembolism. ${ }^{18)}$

The mortality rate in PVD is greater in cases with thrombus compared to pannus and this is related to an emergency condition as a result of hemodynamic deterioration. ${ }^{19)}$ Acute thrombus is less in bileaflet mechanical prosthetic valves. ${ }^{20)}$ However, in our study, PVD was observed in 37 patients with bileaflet mechanical valves.

Thrombolytic therapy can also be administered to patients with valve dysfunction due to thrombus formation. ${ }^{21)}$ In our study, 5 patients underwent thrombolytic treatment preoperatively, however, they were reoperated on because of new thrombus formation or a residual high transvalvular gradient.

Reoperation rates usually do not reflect the real rates of PVD. The real rates can only be estimated by the follow-up of all patients throughout the entire country. This is because in some cases, pannus tissue development is very slow and despite the echocardiographic signs the disease has a subclinical course. In the study of Aoyagi, et al, ${ }^{22)} 54$ patients without PVD symptoms who underwent aortic valve replacement with St. Jude mechanical prosthetic valves were investigated with cineangiography and echocardiography. It was found 16 (29.6\%) of these patients had limited leaflet opening while 5 were reoperated on for a high transvalvular gradient. Of these 5 patients, 4 had pannus and 1 pannus with thrombus.

In conclusion, usually PVD is seen as an emergency condition and hemodynamic deterioration; and thus is responsible for a high prevalence of mortality in valvular reoperations. The results of this study have clearly revealed that left ventricular dysfunction is the major determinant of surgical mortality in patients requiring reoperation for valve dysfunction due to pannus or thrombus. In addition, a lack of cooperation and information exchange between the patient and car- 
diologist plays an important role in the development of thrombus or pannus. This situation is especially true for pregnant patients whose treatment should be changed from coumadinization to heparinization. As shown in the literature, pannus development can be asymptomatic despite the development of a transvalvular gradient. Therefore, all patients should be followed-up by echocardiography and if an obstruction is evident echocardiographically, the patient must be operated on as soon as possible.

\section{REFERENCES}

1. Deviri E, Sareli P, Wisenbaugh T, Cronje SL. Obstruction of mechanical heart valve prostheses: clinical aspects and surgical management. J Am Coll Cardiol 1991; 17: 646-50.

2. Cannegieter SC, Rosendaal FR, Briet E. Thromboembolic and bleeding complications in patients with mechanical heart valve prostheses. Circulation 1994; 89: 635-41. (Review)

3. Thorgeirsson G, Robertson AL Jr. The vascular endothelium-pathobiologic significance. Am J Pathol 1978; 93 : 803-48. (Review)

4. Berenger FP, Cano JP, Rolland PH. Antithrombogenic endothelial cell defense. Basal characteristics in cultured endothelial cells and modulation by short-term and long-term exposure to isosorbide nitrates. Circ Res 1987; 60: 612-20.

5. Horstkotte D. Abnormal cardiac anatomy and physiology. In: Butchart EG, Bodnar E (eds). Thrombosis, Embolism and Bleeding. ICR Publisher, London 1992: 31-69.

6. Horstkotte D, Scharf RE, Schultheiss HP. Intracardiac thrombosis: patient-related and device-related factors. J Heart Valve Dis 1995; 4: 114-20. (Review)

7. Horstkotte D, Burckhardt D. Prosthetic valve thrombosis. J Heart Valv Dis 1995; 4: 141-53. (Review)

8. Martinell J, Jimenez A, Rabago G, Artiz V, Fraile J, Farre J. Mechanical cardiac valve thrombosis. Is thrombectomy justified? Circulation 1991; 84: III 170-5.

9. Teshima H, Hayashida N, Hirohisa Y, et al. Obstruction of St Jude Medical valves in the aortic position: histology and immunohistochemistry of pannus. J Thorac Cardiovasc Surg 2003; 126: 401-7.

10. Davies PF, Remuzzi A, Gordon EJ, Dewey CFJ r, Gimtrone MA Jr. Turbulent fluid shear stress induces vascular endothelial cell turnover in vitro. Proc Natl Acad Sci USA 1986; 83: 2114-7.

11. Sauvage LR, Berger K, Wood SJ. The clinical significance of healing data obtained from study of prosthetic valves in animals. Adv Cardiol 1972; 7: 25-33.

12. Marbarger JP Jr, Clark RE. The clinical life history of explanted prosthetic heart valves. Ann Thorac Surg 1982; 34: 22-33.

13. Durrleman N, Pellerin M, Bouchard D, et al. Prosthetic valve thrombosis: twenty-year experience at the Montreal Heart Institute. J Thorac Cardiovasc Surg 2004; 127: 1388-92.

14. Hinton RC, Kistler JP, Fallon JT, Friedlich AL, Fisher CM. Influence of etiology of atrial fibrillation on incidence of systemic embolism. Am J Cardiol 1977; 40: 509-13.

15. Pitcher I, Curry P. Emboli from prosthetic heart valve during postmenopausal estrogen therapy. Br Med J 1979; 2: $244-5$

16. Staffurth JS, Gibberd MC, Fui SN. Arterial embolism in thyrotoxicosis with artrial fibrillation. Br Med J 1977; 2: 688-90.

17. Wellons HAJr, Strauch RS, Nolan SP, Muller WHJr. Isolated mitral valve replacement with the Kay-Shiley disc valve. Acturial analysis of the long-term results. J Thorac Cardiovasc Surg 1975; 70: 862-8.

18. Kosar F, Gullu H, Sahin I, Aclkgoz N, Topal E, Erdil N. A case of vasospastic angina resulting from coronary emboli in a patient with prosthetic valves. Jpn Heart J 2004; 45: 325-30.

19. Bortolotti U, Milano A, Mossuto E, Mazzaro E, Thiene G, Casarotto D. Early and late outcome after reoperation for prosthetic valve dysfunction: analysis of 549 patients during a 26-year period. J Heart Valve Dis 1994; 3: $81-7$. 
20. Rizzoli G, Guglielmi C, Toscano G, et al. Reoperations for acute prosthetic thrombosis and pannus: an assessment of rates, relationship and risk. Eur J Cardiothorac Surg 1999; 16: 74-80.

21. Ozkan M, Kaymaz C, Kirma C, et al. Intravenous thrombolytic treatment of mechanical prosthetic valve thrombosis: a study using serial transesophageal echocardiography. J Am Coll Cardiol 2000; 35: 1881-9.

22. Aoyagi S, Nishimi M, Kawano H, et al. Obstruction of St Jude Medical valves in the aortic position: significance of a combination of cineradiography and echocardiography. J Thorac Cardiovasc Surg 2000; 120: 142-7. 